\title{
Breast Filariasis Diagnosed by Real Time Sonographic Imaging: A Case Report
}

\author{
Poonam Sherwani, ${ }^{1, *}$ Shweta Singhal, Nidhi Kumar, Mahender Kaur Narula, ${ }^{1}$ Rama Anand, \\ and Om Prakash Pathania ${ }^{2}$ \\ ${ }_{1}^{1}$ Department of Radiodiagnosis, Lady Hardinge Medical College, Smt Sucheta Kriplani Hospital, New Delhi, India \\ ${ }^{2}$ Department of Surgery, Lady Hardinge Medical College, Smt Sucheta Kriplani Hospital, New Delhi, India \\ ${ }^{*}$ Corresponding author: Poonam Sherwani, Department of Radiodiagnosis, Lady Hardinge Medical College, Smt Sucheta Kriplani Hospital, New Delhi, India. Tel: +91-9540464879, \\ E-mail:poonam_doc2003@yahoo.co.in \\ Received 2014 April 23; Revised 2014 June 15; Accepted 2014 June 24.
}

\begin{abstract}
A 30-year-old woman presented with a palpable subcutaneous nodule in the areolar region of the left breast. Sonomammographic examination revealed 2 cystic lesions showing typical "filarial dance" as vigorous twirling movement of multiple curvilinear echoes with mixed red blue color Doppler signals that was non-rhythmic, nonpulsatile, and the characteristic pulse Doppler trace due to irregular worm movement. Real time sonographic demonstration of these typical features is pathognomonic for filariasis, especially in endemic areas and treatment should be initiated without delay on the basis of ultrasound.
\end{abstract}

Keywords: Filariasis, Eosinophilia, Ultrasound, Worms

\section{Introduction}

Filariasis is frequently encountered in Asian, African and some of the South American countries. Breast filariasis has been reported in the Indian subcontinent where the organism is endemic. Lymphatic filariasis in humans is commonly caused by Wuchereria bancrofti and Brugia malayi. A large majority of the cases found in India are attributed to infection by Wuchereria bancrofti, which mainly affects the lymph nodes and the lymphatic channels. The breast is an uncommon site of involvement.

\section{Case Presentation}

We present a 30-year-old female who presented in surgical out patient department with complaints of palpable subcutaneous nodular swelling in the areolar region of the left breast for past 2 months with on and off pain, and itching and one episode of serous discharge. The overlying and adjacent skin appeared normal (Figure 1). On examination, there was no palpable lump in the breast and no palpable axillary lymphadenopathy.

Sonomammography was performed using a high frequency transducer of 5-17 MHz (Philips IU-22) that revealed a $6 \times 3 \mathrm{~mm}$ cystic lesion containing numerous curvilinear echoes with 2 parallel walls and an anechoic central lumen, exhibiting vigorous twirling movements suggestive of "filarial dance" in the subcutaneous plane in the areolar region of the left breast. Another $2 \times 2 \mathrm{~mm}$ cystic lesion was noted deep to the above-mentioned cyst with a tubular channel interconnecting the two cysts. The cystic le- sion also showed similar curvilinear echoes with vigorous twirling movement (Video 1). Few other tubular channels were seen adjacent to these cysts that were not converging towards the nipple and the cysts were presumed to represent focally distended lymphatic ducts (Figure 2A and B).

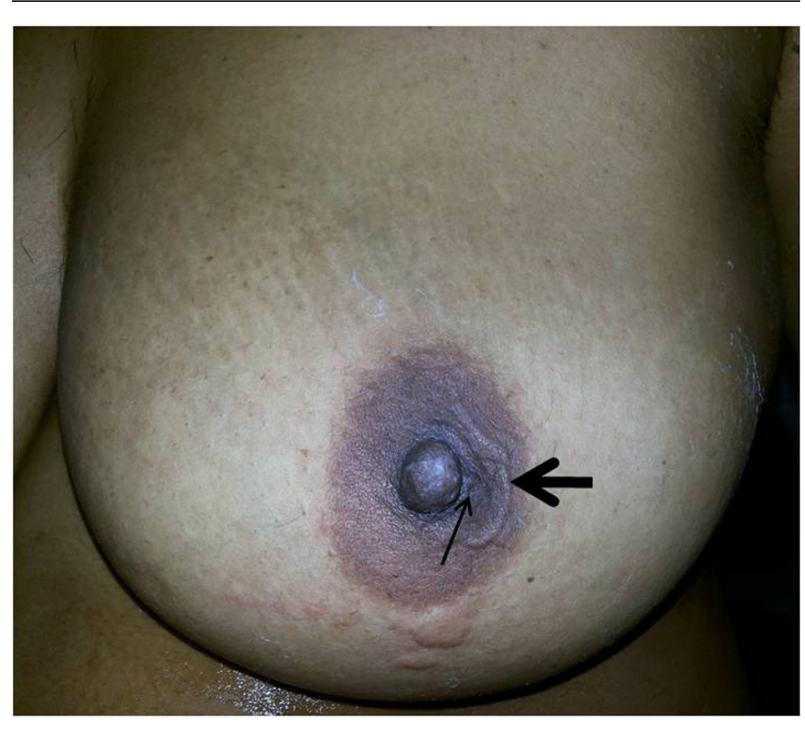

Figure 1. A 30-year-old female with palpable subcutaneous nodular swelling in the areola from 2 months ago. Local examination shows a nodular swelling (line arrow) with adjacent cord like swelling (solid arrow) in the areolar region of the left breast.

Copyright (C) 2016, Tehran University of Medical Sciences and Iranian Society of Radiology. This is an open-access article distributed under the terms of the Creative Commons Attribution-NonCommercial 4.0 International License (http://creativecommons.org/licenses/by-nc/4.0/) which permits copy and redistribute the material just in noncommercial usages, provided the original work is properly cited. 
Real time sonogram shows characteristic vigorous twirling movement suggestive of "filarial dance".

Color Doppler examination of the lesion revealed red, blue, and mixed color Doppler signals that were non-rhythmic, non-pulsatile, and rapidly changed in size and position (Figure 3A). Spectral Doppler trace showed the characteristic pattern of irregular worm movement (Figure 3B).

Fine needle aspiration of the cyst containing moving echoes yielded a straw-colored fluid and microscopic examination revealed the presence bancroftian microfilaria. There was eosinophilia in the blood; however, the peripheral blood smear did not reveal any hemoparasite. The patient was treated with a regimen of diethylcarbamazine citrate $100 \mathrm{mg}$ thrice daily for 2 weeks with a 2-week drug-free interval between each course for a period of 3 months. The follow-up ultrasound that was performed after 2 weeks of treatment showed prominent lymphatic channels in the subcutaneous tissue and significant reduction in the dilatation of the deep lymphatic channels. Twirling movement of the worms was no longer seen that was suggestive of dead worms (Figure $4 \mathrm{~A}$ and $\mathrm{B}$ ).
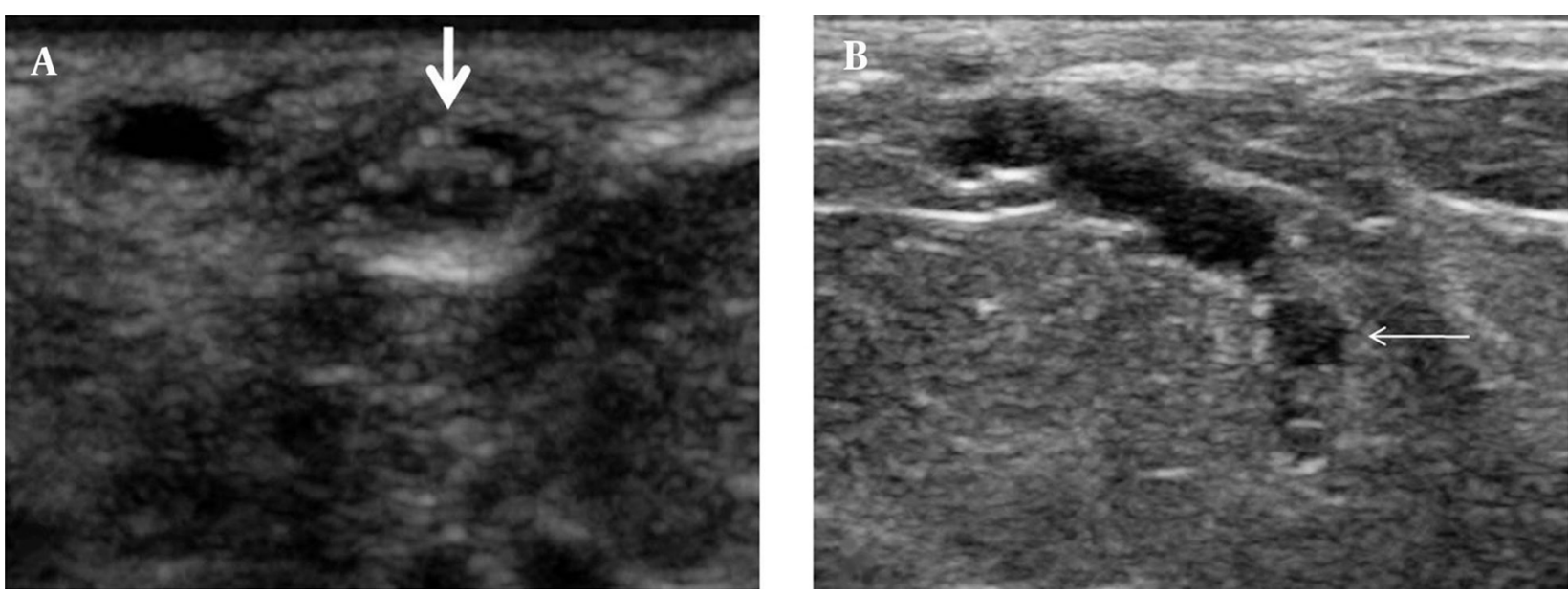

Figure 2. Sonography of the left breast shows two cystic lesions, one in the subcutaneous plane (solid arrow) (A) and the other in the fatty breast parenchyma (line arrow) (B), both showing multiple curvilinear echoes with a dilated interconnecting tubular channel.
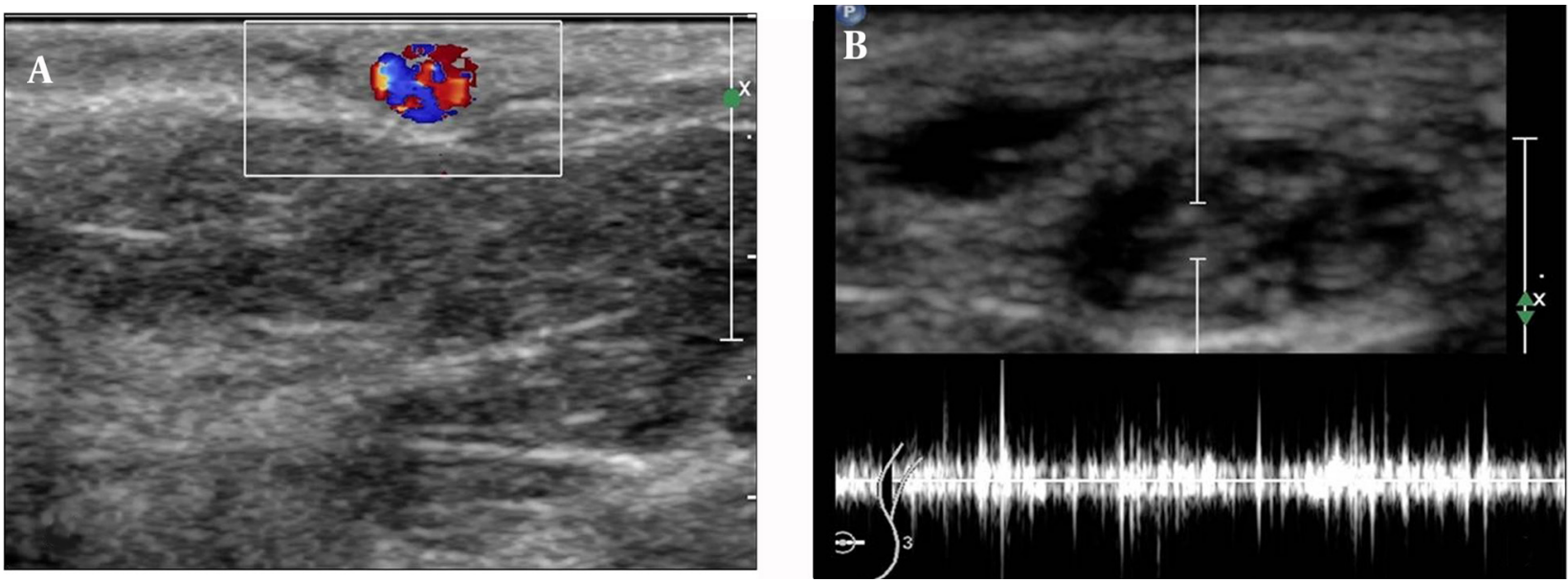

Figure 3. A, Color Doppler sonogram shows mixed color Doppler signals due to color motion artifact. B, Spectral trace shows irregular pattern of worm movement. 

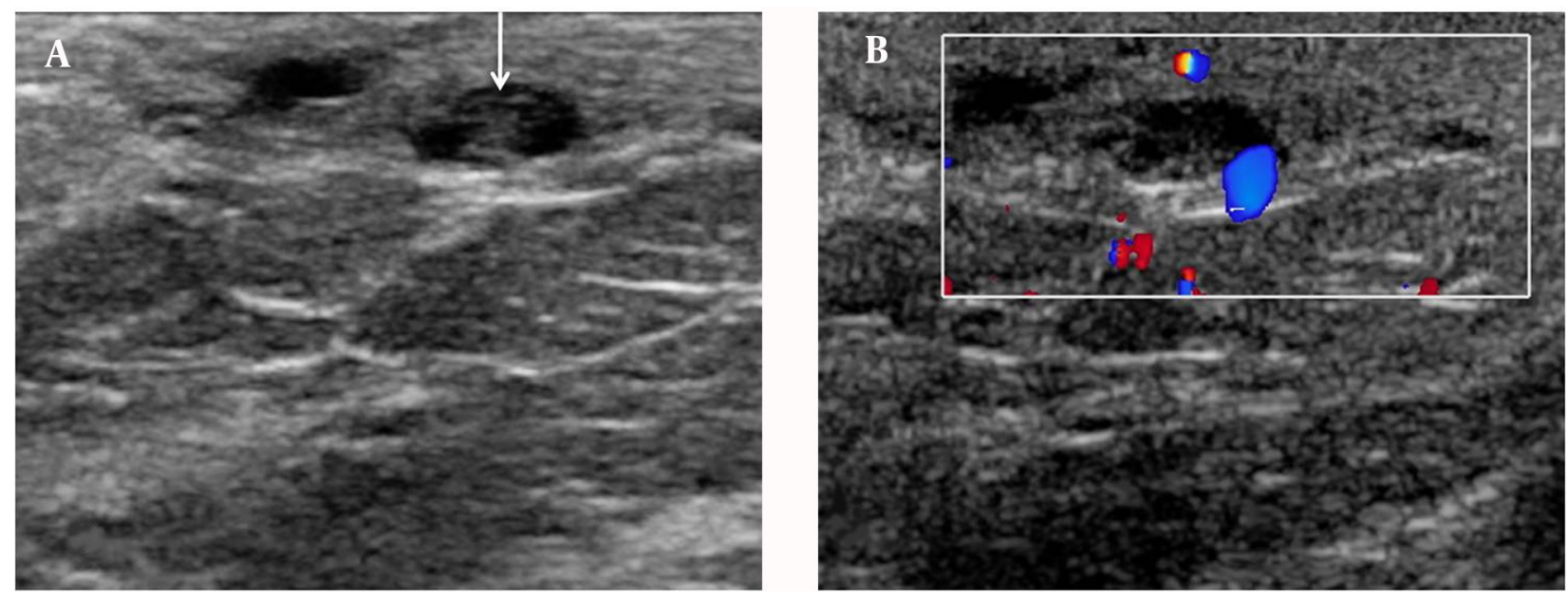

Figure 4. A, Follow-up ultrasound showed prominence of lymphatic channels with linear echoes (arrow), and loss of twirling movement (dead worms). $\mathrm{B}$, the color motion artifact was not seen.

Video 1: Real time sonogram shows characteristic vigorous tworling movement suggestive of "filarial dance"

\section{Discussion}

In India, Wuchereria bancrofti is a public health problem. It is endemic all over India, except in Jammu and Kashmir, Himachal Pradesh, Haryana, Rajasthan, and the eastern states. It is distributed chiefly along the seacoast and along the banks of big rivers (1). Adult worms are found in the lymphatic vessels and lymph nodes of human beings only; therefore, bancroftian filariasis is not a zoonotic disease.

In lymphatic filariasis, the adult parasites live in the lymphatic vessels whilst their offspring, the microfilariae circulate in the peripheral blood and are available to the mosquito vectors when they feed on the human blood (2). In lymphatic filariasis, the sites commonly involved are bronchial aspirate, cervico-vaginal smears and pleural and pericardial fluids (3). Breast lesions, though unusual, are not rare and swelling of the breast due to obstruction of the dermal lymphatics by the filarial worm has been documented (4).

Lymphatic vessels of the breast get infested with the larvae, which causes lymphangitis, fibrosis, and lymphatic obstruction (5). The patient usually presents with non-tender swelling; the most common site being the upper outer quadrant (2). Our patient presented with small non-tender subcutaneous swelling in the left areolar region with on and off itching. However, changes of peau d'orange and axillary lymphadenopathy have also been reported (6).

Imaging plays an important role in the diagnosis. Ultrasound is a valuable tool as real time ultrasound demonstrates the typical vigorous movement of the filarial worms termed as "filarial dance" which is classical.

Amaral et al. (7) first reported the real-time sonographic visualization of adult W.bancrofti in the scrotum of infected men, naming it the "filarial dance sign". Dreyer and colleagues first demonstrated the filarial dance in the breast (8). Rathi et al. (9) described the color motion artifact, which is produced by the swirling motion of the parasite. In our case also, typical filarial dance in the cystic lesion that was presumed to be a focally dilated lymphatic duct with living microfilarial worms showing color motion artifact was seen. This was confirmed on cytologic examination of the aspirate. These findings were similar to those of Surendrababu et al. (10).

To conclude, our case highlights the importance of sonomammography in the diagnosis of palpable and non palpable breast nodules in filarial endemic areas.

\section{Acknowledgments}

To the surgery department faculty and the post graduates.

\section{Authors' Contributions}

Study concept and design: Poonam Sherwani. Aquisition of data: Shweta Singhal. Analysis and interpretation of data: Mahender Kaur Narula. Drafting of the manuscript: Poonam Sherwani. Critical revision of the manuscript for important intellectual content: Mahender Kaur Narula. Statistical analysis: Nidhi Kumar. Administrative, technical, and material support: Om Prakash Pathania. Study supervision: Mahender Kaur Narula. Language and grammar edition: Rama Anand.

\section{Financial Disclosure}

Poonam Sherwani and other co-authors have no financial interest in the material of the manuscript.

\section{References}

1. Park K. Epidemiology of communicable diseases. Park's Textbook of Preventive and Social Medicine. 22 ed. Jabalpur: Banarsidas Bha- 
not; 2013. pp. 245-6.

2. Upadhyaya V, Upadhyaya DN, Sarkar S. An interesting case of breast filariasis. Indian J Radiol Imag. 2006;16(4):915-7.

3. Sahu KK, Pai P, Raghuveer CV, Pai RR. Microfilaria in a fine needle aspirate from the salivary gland. Acta Cytol. 1997;41(3):954

4. Singh NG, Chatterjee L. Filariasis of the breast, diagnosed by fine needle aspiration cytology. Ann Saudi Med. 2009;29(5):414-5.

5. Alkadhi H, Garzoli E. Images in clinical medicine. Calcified filariasis of the breasts. N Engl J Med. 2005;352(2):e2.

6. Lahiri VL. Microfilariae in nipple secretion. Acta Cytol. 1975;19(2):154

7. Amaral F, Dreyer G, Figueredo-Silva J, Noroes J, Cavalcanti A,
Samico SC, et al. Live adult worms detected by ultrasonography in human Bancroftian filariasis. Am J Trop Med Hyg. 1994;50(6):753-7.

8. Dreyer G, Amaral F, Noroes J, Medeiros Z. Ultrasonographic evi dence for stability of adult worm location in bancroftian filariasis. Trans R Soc Trop Med Hyg. 1994;88(5):558.

9. Rathi V, Bhargava SK, Gupta A, Jain S. Filarial dance in a breast mass on colour Doppler imaging. Australas Radiol. 2006;50(2):183-5.

10. Surendrababu NR, Thomas E, Rajinikanth J, Keshava SN. Breast filariasis: real-time sonographic imaging of the filarial dance. $J$ Clin Ultrasound. 2008;36(9):567-9. 